\section{Atypische Frakturen: Warum Bisphosphonate (manchmal) den Knochen zerbrechlich machen}

Bisphosphonate und auch Denosumab vermindern die Knochenresorption, was bei einer längeren Anwendung die Knochendichte erhöht, aber vor allem auch bei längerer Anwendung und bei höheren Dosen eine verminderte sogenannte "Bruchzähigkeit" zur Folge haben kann. Dies wird in einer Studie in den Proceedings of the National Academy of Sciences für das Auftreten von atypischen Femurfrakturen verantwortlich gemacht, die als seltene, aber folgenreiche Nebenwirkung der genannten Osteoporose-Medikamente auftreten können.

Bruch- oder Risszähigkeit ( „Fracture Toughness") ist ein Begriff aus der Bruchmechanik, die sich mit den Gründen für die Bildung von Rissen in Materialien beschäftigt. Die "Fracture Toughness" wird mit sogenannten R-Kurven bestimmt. Sie messen die Länge der Risse (x-Achse), die sich unter der Einwirkung äußerer Kräfte (y-Achse) bilden. Eine steile R-Kurve zeigt eine verminderte "Fracture Toughness" an.

Ein Team um Eve Donnelly von der Cornell Universität in New York hat die R-Kurve von Knochenspänen bestimmt. Die Späne waren bei 33 Patientinnen bei einer Operation zur Behandlung einer Femurfraktur oder bei der Implantation eines Kunstgelenks entnommen worden. Darunter waren auch zwölf Frauen, die nach der Einnahme von Bisphosphonaten (über eine mittlere Dauer von 8,2 Jahren) eine atypische Femurfraktur erlitten hatten.

Atypische Femurfrakturen sind eine erst vor einigen Jahren entdeckte Komplikation, zu der es unter der Langzeiteinnahme von Bisphosphonaten kommen kann - was die USArzneimittelbehörde zu der Empfehlung veranlasste, die Medikamente nach drei bis fünf Jahren abzusetzen. Die betroffenen Frauen hatten demnach die Medikamente nach heutigem Verständnis viel zu lange eingenommen.

Um die R-Kurve zu bestimmen, wurden Knochenspäne in der Größe von $5 \mathrm{~mm} \times$ $0,5 \mathrm{~mm} \times 0,5 \mathrm{~mm}$ einem Biegungsstress ausgesetzt, während die Rissbildung und Ausdehnung in einem Rasterelektronenmikroskop beobachtet wurde. Die Untersuchungen ergaben eine deutlich steilere R-Kurve für Knochenspäne der Patientinnen, die eine atypische Fraktur erlitten hatten, gegenüber Frauen mit typischen Femurfrakturen oder auch Frauen ohne
Frakturen, bei denen ein Gelenkaustausch vorgenommen wurde.

Interessant war auch der Verlauf der Materialrisse im Knochen. Bei knochengesunden Frauen oder Patienten mit typischen Frakturen sind die Risse häufig gewunden (erhöhte "Turtuosität"), während sie bei den Frauen mit atypischen Femurfrakturen oft den direkten Weg durch das Knochengewebe nehmen. Dies könnte die röntgenologischen Befunde erklä-

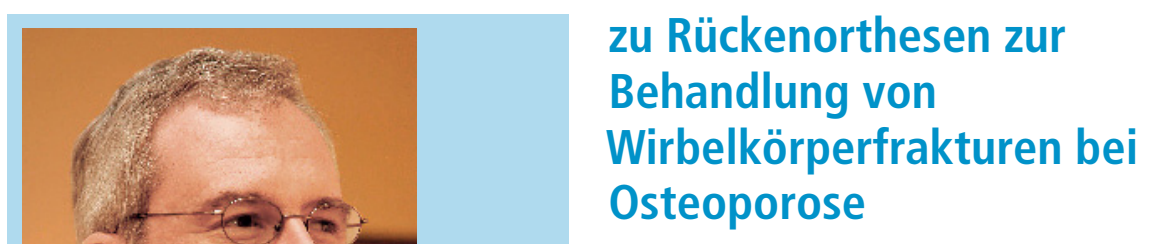

Rückenorthesen zur Behandlung von Wirbelkörperfrakturen bei Osteoporose haben sich inzwischen auch international durchgesetzt. Alle im Folgenden dargestellten Studien wurden unabhängig von Herstellerinteressen durchgeführt, sodass Interessenkonflikte weitgehend ausgeschlossen werden können.

\section{Akute Schmerzlinderung bei Osteoporose-assoziierten Kompressionsfrakturen}

So berichten beispielsweise Rzewuska M. et al. aus Sydney, Australien, im Rahmen eines systematischen Reviews mit Metaanalyse über die Wirksamkeit einer konservativen Behandlung bei Osteoporose-assoziierten Kompressionsfrakturen auf eine akute Schmerzlinderung. In fünf RCTs (318 Frauen; 32 Männer; Alter zwischen 66,7 und 81,5 Jahren) wurden die Patienten nach frischer Wirbelkörperfrak- 
tur entweder mit medikamentöser Schmerztherapie (Opioide, nichtsteroidale Antirheumatika, transdermales Lidocain), transkutane Stimulation der Nerven sowie Bettruhe und Orthesen versorgt. Ausgeschlossen wurden Wirbelkörperfrakturen, die entweder traumatisch oder durch Metastasen verursacht worden waren. Die Schmerzintensität wurde mittels VAS oder der Miltner-Skala bestimmt, an sekundären Endpunkten funktionelle Einschränkungen, Parameter der Lebensqualität und Nebenwirkungen dokumentiert.

Zusammenfassend wurden fünf RCTs $(n=350)$ eingeschlossen (darunter eine placebokontrollierte Studie). Vier kontrollierte Studien untersuchten Schmerzmedikation (zwei Studien) und zwei Studien Rückenorthesen. Der PEDro-Score befand sich zwischen 4 und 7, sodass von einer eher niedrigen Evidenzlage auszugehen ist. In zwei Studien konnte durch den Einsatz der Rückenorthesen eine signifikante Schmerzreduktion von $-1,47$ (95\% KI $\left.-1,82 ;-1,13 ; 1^{2}=0 \%\right)$ und zusätzlich eine ebenfalls signifikante Verminderung der funktionellen Einschränkungen von -1,73 (95\% KI $\left.-2,09 ;-1,37 ; I^{2}=0 \%\right)$ festgestellt werden. Schnelle und Kurzzeiteffekte wurden durch Diclofenac und Tramadol im Vergleich zu chinesischer Medizin beobachtet. Oxycodon und Tapentadol hatten keinen signifkanten Einfluss.

Quelle: Rzewuska M et al. The efficacy of conservative treatment of osteoporotic compression fractures on acute pain relief: a systemic review with metaanalysis. Eur Spine J 2015; 24: 702-714.

Evidenzlevel 1++

\section{Rückenorthesen zur Behandlung von Osteoporose und von Wirbel- körperfrakturen}

Newman M. et al. untersuchten in einem systematischen Review Rückenorthesen zur Behandlung von Osteoporose der Wirbelsäule und Wirbelkörperfrakturen bei Osteoporose. Acht RCTs und vier nicht randomisierte Studien wurden berücksichtigt. Eingeschlossen wurden Erwachsene mit der Diagnose einer Osteoporose der Wirbelsäule oder einer Osteopenie mit und ohne Wirbelkörperfrakturen. Behandelt wurde mit Rückenorthesen mit oder ohne medizinischer Trainingstherapie. Eingesetzt wurden starre Thorakolumbalorthesen, halbstarre Kyphoorthesen und flexible Lumbosakralorthesen. Zu den Studienendpunkten zählten Körperhaltung nach stattgehabter Wirbelkörperfraktur, Schmerzen, Rückenmuskelkraft und Ausdauer, Rückenverformungen und Körpergröße sowie funktionelle Einschränkungen und Parameter der Lebensqualität. Sekundäre Evaluationsparameter waren Nebenwirkungen und Compliance der Behandlung.

Bei starren Rückenorthesen, die in drei Studien $(n=153)$ nach akuter Wirbelkörperfraktur eingesetzt wurden, war die Komplikationsrate am höchsten. Es gab keine Evidenz dafür, dass mit Rückenorthesen die Verformungen einzelner Wirbelkörper beeinflusst werden könnten. Neun Studien $(n=473)$ von unterschiedlicher Qualität untersuchten Orthesen in der subakuten und Rehabilitationsphase. Davon lassen drei Studien mit Thorakolumbalorthesen, die nach dem halbstarren Rucksackprinzip funktionieren, einen Nutzen hinsichtlich Rumpfmuskelkraft, Schmerzen, Körperhaltung und Parameter der Lebensqualität vermuten. Eine Studie, die mit einer Orthese nach dem Rucksackprinzip und zusätzlichen Gewichten durchgeführt wurde, verbesserte das Gleichgewicht.

Quelle: Newman M et al. Spinal orthoses for vertebral osteoporosis and osteoporotic vertebral fracture: a systemic review. Arch Phys Med Rehabil 2016; 97: 1013-1025.

\section{Evidenzgrad 2++}

Vergleich einer starren 3-Punkt-Orthese mit einer flexiblen Rückenorthese nach dem Rucksackprinzip

Meccariello L. et al. aus Siena, Italien, führten einen RCT zum Vergleich einer starren 3-Punkt-Orthese mit einer flexiblen und dynamischen Rückenorthese nach dem Rucksackprinzip (Spinomed ${ }^{\circledR}$ ) durch. 140 Patienten im Alter zwischen 65 und 93 Jahren (100 Frauen, 40 Männer, $\varnothing 82,3$ J.) mit einer Wirbelkörperfraktur bei Osteoporose zwischen Th6 und L3 und akuten Rückenschmerzen erhielten konsekutiv entweder eine 3-Punkt-Orthese oder eine flexible, dynamische Rückenorthese (Spinomed $\left.^{\circledR}\right)$. Zu Beginn und bei der Nachuntersuchung nach einem, drei und sechs Monaten wurden Schmerzen mittels VAS und funktionelle Einschränkungen mit dem "Oswestry Low Back Pain Diasability Questionnaire" erhoben. Die Schwere der Fraktur wurde entsprechend der Klassifikation von Genant dokumentiert. Nach einer Studiendauer von sechs Monaten kam es in der Gruppe der 3-Punkt-Orthese bei 28 Patienten und in der Spinomed ${ }^{\circledR}$-Gruppe bei acht Patienten zu schwerwiegenden Nebenwirkungen. Unter anderem waren in der 3-Punkt-OrthesenGruppe sechs neue Frakturen und in der Spinomed $^{\circledR}$-Gruppe zwei neue Frakturen aufgetreten, was statistisch signifikant war $(p<0,05)$. Schließlich war es nach sechs Monaten zu einer Verbesserung der Lungenfunktion anhand des $\mathrm{FEV}_{1}$ gekommen. Zusammenfassend kam es in der Spinomed ${ }^{\circledR}$-Gruppe nach sechs Monaten Tragezeit zu einer besseren Schmerzkontrolle und einer verbesserten Lungenfunktion im Vergleich zur 3-PunktOrthese.

Quelle: Meccariello $L$ et al. Dynamic corset versus three-point brace in the treatment of osteoporotic compression fractures of the thoracic and lumbar spine: a prospective, comparative study. Aging Clin Exp Res 2017;29:443-449.

\section{Evidenzgrad 1-}

\section{Interessenkonflikt}

MP erhält Honorare für Vorträge und Beratung durch die Firma medi GmbH \& Co. KG, Bayreuth. 